Trade Liberalization, Heterogeneous Firms and the Soft Budget Constraint

by

\author{
Michael Alexeev* \\ and
}

Yong Joon Jang**

June 2010

* Department of Economics, Indiana University, Bloomington, IN 47405; e-mail: malexeev@indiana.edu

** Korea Institute for International Economic Policy (KIEP), Seoul 137-747, Korea, email: yjjang@umail.iu.edu

We are grateful to Andrei Levchenko and the anonymous referee for their insightful comments. All remaining errors are our responsibility. 


\begin{abstract}
We analyze the interaction between the soft budget constraint (SBC) and international trade by placing Segal's (1998) SBC model within Melitz's (2003) framework of international trade with heterogeneous monopolistically competitive firms. As in Segal's model, SBC may result in moral hazard. The opening to international trade adds another sort of inefficiency. Some firms that would have become exporters in the absence of SBC choose to apply low effort and not export in order to extract a subsidy from the government. This effect takes place when the trade costs are sufficiently low. Overall, however, trade liberalization reduces inefficiencies generated by SBC. The number of firms subject to moral hazard SBC decreases, aggregate effort level increases and aggregate profits lost due to SBC-induced sub-optimal effort decline as trade costs decrease.
\end{abstract}

JEL Classification: F12, D21, H25

Keywords: Soft budget constraint, international trade, heterogeneous firms, monopolistic competition 


\section{Trade Liberalization, Heterogeneous Firms and the Soft Budget Constraint}

\section{Introduction}

A firm's budget constraint is said to be "soft" if an unprofitable firm can count on being bailed out by the state. ${ }^{1}$ The term Soft Budget Constraint (SBC) initially referred to enterprises in centrally planned economies (see Kornai, 1979, 1980). Since then, however, the SBC concept has received much wider attention in economics literature (see surveys by Maskin and Xu, 2001, and Kornai et al., 2003). While SBC has been most often observed in the socialist economies, the economies in transition, and developing countries, it has recently become highly relevant to established market economies, including the U.S. where the federal government has bailed out several financial firms and two major automobile manufacturers.

One important issue in the SBC literature has been the determination of factors that weaken government incentives to bail out insolvent firms. For example, Dewatripont and Maskin (1995) and Alexeev and Kim (2004) showed that decentralized lending market hardens budget constraints. Also, Segal (1998) showed that while government subsidy can be a subgame perfect equilibrium in a game between the benevolent government and a monopoly, competition among homogeneous firms could eliminate SBC under certain conditions. In this paper we consider a market with monopolistically competitive firms and examine the effect of trade liberalization on SBC. Our setup incorporates the main elements of Segal's model within Melitz's (2003) framework of international trade. In Segal's benchmark model, the possibility of receiving a subsidy creates a moral hazard for a monopoly that may decide to apply lower than socially optimal effort in order to make it sub-game optimal for the government to provide the subsidy. In

\footnotetext{
${ }^{1}$ This rather loose definition is taken from Segal (1998).
} 
our model, the firms are monopolistic competitors with heterogeneous productivities. Thus, ours is the first SBC model that incorporates both moral hazard and heterogeneous firms in a monopolistically competitive environment. In addition, this is the first model that analyzes the relationships between SBC and international trade, including the firm's propensity to export.

We find that if trade costs are sufficiently low, SBC eliminates incentives to export among some firms that would have exported in the absence of SBC. Perhaps more importantly, we show that for any trade costs level, trade liberalization reduces SBC-induces inefficiencies in the economy. The main inefficiency arises because some firms subject to SBC apply less than socially optimal effort. We show that this loss declines in trade costs. In addition, we show that social loss measured as the amount of profits lost by the firms due to lower effort also decreases in trade costs. Finally, we demonstrate that the inefficiency resulting from loss-making firms being kept afloat by government subsidies diminishes as trade costs decline.

The rest of the paper is organized as follows. In Section 2 we set up the basic model with heterogeneous firms' moral hazard in monopolistic competition under autarky. In Section 3 we extend the basic model to the open economy, and analyze the effects of lower trade costs on SBC. Section 4 concludes.

\section{A model of SBC under Monopolistic Competition and Autarky}

\subsection{Demand}

Consider an economy populated by homogeneous consumers and heterogeneous firms. A representative consumer has income $I$ and CES preferences over a set of differentiated goods indexed by $x \in X$, where $X$ is a set of all potentially available goods. Consumer income consists 
of wages normalized to 1 paid for inelastically supplied labor, $L$, and firm profits, $\pi$, which are equally distributed among all consumers. Consumer optimization problem is:

$$
\begin{aligned}
& \qquad \max _{q(x)} U=\left[\int_{x \in X} q(x)^{\rho} d x\right]^{\frac{1}{\rho}}, \quad 0<\rho<1 \\
& \text { s.t. } \int_{x \in X} p(x) q(x) d x=I=L+\pi
\end{aligned}
$$

where $q(x)$ is the demand for $\operatorname{good} x, p(x)$ is the price of $x, \sigma$ is the elasticity of substitution between any two goods with $\sigma>1$ and $=\frac{\sigma-1}{\sigma}$. We define the aggregate price index $P$ as

$$
P=\left[\int_{x \in X} p(x)^{1-\sigma} d x\right]^{\frac{1}{1-\sigma}}
$$

The demand for good $x$ is derived from the consumer maximization problem:

$$
q(x)=\left(\frac{I}{P}\right)\left[\frac{p(x)}{P}\right]^{-\sigma}
$$

and the price elasticity of demand is:

$$
\varepsilon_{p}=-\frac{d q}{d p} \frac{p}{q}=\sigma
$$

\subsection{Production}

We consider a monopolistically competitive market consisting of $N$ firms (not all of which choose to operate) in which each firm produces a different good (variety) $x$ using increasing returns technology defined in (6) below via the cost function. The only factors of production are labor and "effort." A firm chooses its effort level $a(1 \leq a \leq A)$ after entry to reduce its 
marginal cost, $M C$. Following Segal (1998), we assume that the firm incurs no cost for its effort, and only the firm knows its effort level, so that no contracts based on the level of effort are possible. $^{2}$

Firm's effort reduces $M C$ in a multiplicative fashion, and the total cost for each firm applying effort $a$ is given by:

$$
T C(\theta, a)=f+\left[\frac{1}{\theta a}\right] q
$$

where $f>0$ is fixed cost of production which is the same for all firms. ${ }^{3}$ After finding out its productivity $\theta \geq 1$ the firm decides whether to produce or not. We assume that the firm's "effort-inclusive" productivity $a \theta$ is observable.

Each firm draws its productivity from a Pareto distribution with the cumulative distribution function:

$$
F(\theta)=1-\theta^{-\gamma}, \quad \gamma>\operatorname{Max}\{1, \sigma-1\}
$$

(The assumption that $\gamma>\operatorname{Max}\{1, \sigma-1\}$ assures that in equilibrium the size distribution of firms has a finite mean.)

The firm's profit maximization problem is:

$$
\begin{gathered}
\max _{p} \pi(\theta, a)=p q-\frac{1}{\theta a} q-f, \\
\text { subject to } \pi(\cdot) \geq 0
\end{gathered}
$$

\footnotetext{
${ }^{2}$ Note that $a$ can denote some other type of investment that reduces marginal cost of production. The assumption that effort is costless simplifies exposition while producing particularly stark results. The qualitative nature of the results would not change if effort were assumed to be costly.

${ }^{3}$ One can argue that firms subject to SBC often have higher fixed costs than other firms. As we discuss later, the assumption of identical fixed costs is easily relaxed.
} 
implying that the equilibrium price, output and profit of each firm are, respectively:

$$
\begin{aligned}
& p(\theta, a)=\frac{1}{\rho \theta a} \\
& q(\theta, a)=I P^{\sigma-1}(\rho \theta a)^{\sigma} \\
& \pi(\theta, a)=\frac{I(\rho \theta a P)^{\sigma-1}}{\sigma}-f
\end{aligned}
$$

Clearly, without subsidies, every firm that chooses to operate will apply maximum effort, $A$, because $\pi(\theta, A)>\pi(\theta, a)$ for all $a<A$. Also, there is a cut-off level $\bar{\theta}_{A}$ of productivity which satisfies $\pi\left(\bar{\theta}_{A}, a\right)=0$ implying that $\bar{\theta}_{A}$ is:

$$
\bar{\theta}_{A}=\frac{1}{\rho A P}\left(\frac{f \sigma}{I}\right)^{\frac{1}{\sigma-1}}
$$

A firm with productivity $\theta<\bar{\theta}_{A}$ will decide not to produce while a firm with $\theta \geq \bar{\theta}_{A}$ will operate. Recalling that the measure of firms that can potentially operate is $N$ and using (7), (9), and (12), we can obtain the aggregate price index:

$$
\begin{gathered}
P=\left(N \int_{\bar{\theta}_{A}}^{\infty} p(\theta, A)^{1-\sigma} d F(\theta)\right)^{\frac{1}{1-\sigma}}=\left(N \int_{\bar{\theta}_{A}}^{\infty}\left(\frac{1}{\rho \theta A}\right)^{1-\sigma} \gamma \theta^{-\gamma-1} d \theta\right)^{\frac{1}{1-\sigma}} \\
\Rightarrow P=P\left(\bar{\theta}_{A}\right)=\left(\frac{\gamma-(\sigma-1)}{\left(\frac{(\sigma-1) A}{\sigma}\right)^{\sigma-1} N \gamma\left(\bar{\theta}_{A}\right)^{\sigma-1-\gamma}}\right)^{\frac{1}{\sigma-1}} \\
\Rightarrow P=\left(\frac{\lambda}{N \gamma(\rho A)^{\gamma}\left(\frac{\sigma f}{I}\right)^{\beta}}\right)^{\frac{1}{\gamma}}
\end{gathered}
$$


where $\beta=1-\frac{\gamma}{\sigma-1}, \lambda=\gamma-(\sigma-1)$.

\subsection{Introduction of Government Subsidy}

Following Dewatripont and Maskin (1995) and Segal (1998) we view SBC as a result of government's inability to precommit to not subsidizing a firm that would go out of business without the subsidy, but would continue operating with the subsidy. Suppose that a benevolent government decides to support a subset of firms by providing a subsidy, $s>0$, if the firms are going to exit the market without such support. ${ }^{4} \mathrm{We}$ assume that only relatively few of the total number of firms, specifically, $N_{s}<<$ firms, might be eligible for government support, so that the choices of these firms do not significantly affect the aggregate price index and aggregate profit in the economy. ${ }^{5}$ These might be firms in particular industries that the government deems worthy of subsidizing if they are on the verge of exiting. The distribution of productivities of these firms is assumed to be the same as for all firms. In the rest of the paper, we will refer to these firms either as "eligible for subsidy" or as "potentially SBC firms." In principle, we do not need to separate firms eligible for subsidy from other firms. All we need for our model to remain tractable and for our results to hold is that the aggregate price index and profit are (approximately) independent of the number of subsidized firms. We also assume that the

\footnotetext{
${ }^{4}$ In Segal (1998) the size of the subsidy is endogenous and is determined based on consumer surplus generated by the subsidized firm and the government's cost of raising funds. In order to keep our model tractable, we make the subsidy exogenous. However, the subsidy is provided only if the firm can credibly threaten to go out of business unless the subsidy is given.

${ }^{5}$ As will be seen later, the presence of the subsidy to some firms implies that some firms with productivities below $\bar{\theta}_{A}$ would choose to operate and also some of the firms with productivities above $\bar{\theta}_{A}$ would not apply maximum amount of effort. If there were many such firms, they would affect the aggregate price index and the aggregate profit in the economy, The change in the price index due to non-maximum effort by firms makes the problem intractable. The changes in the aggregate profit and price index due to the presence of firms that would not have operated at all without a subsidy makes already complicated calculations substantially more tedious, but does not affect the results as long as these low productivity firms do not affect the difference between profits and price indices under autarky and in the open economy. A similar assumption of the negligible effect of part of the economy on the aggregate price index is present, for example, in Chaney (2005) and in Do and Levchenko (2009).
} 
government obtains funds for its expenditures, including the subsidy from taxing labor income, so that consumer income $I$ introduced above is after-tax income. ${ }^{6}$ In addition, we assume that

$$
0 \leq s<f
$$

As Segal (1998) showed, even though effort is costless, the possibility of obtaining a subsidy might induce some firms not to exert maximum possible effort. This happens because low effort can serve as a precommitment to not producing without a subsidy. After a firm that is in principle eligible for a subsidy enters the market and observes its productivity $\theta$, it bargains with the government for the subsidy via a game with the following timing:

- Stage 1: The firm chooses its effort level $a$.

- Stage 2: The firm asks the government for subsidy, $s$, threatening to stop production without $s$.

- Stage 3: The government, observing the firm's effort-inclusive productivity a $\theta$ (but neither $\theta$ nor $a$ separately) decides whether to accept or reject the firm's offer.

- Stage 4: If the offer is accepted, the firm obtains the subsidy and produces at its profit maximizing level, given its productivity $\theta$ and effort $a$. Otherwise, the firm decides whether to produce or not.

By considering firms' heterogeneous productivity and the game above simultaneously, we obtain the following three propositions which describe the relationship between SBC and the

\footnotetext{
${ }^{6}$ We assume that if subsidy amounts change, the government offsets these changes by changing its other expenditures on items that do not directly affect production such as improving environment or providing foreign aid.
} 
productivity distribution of firms in the market. ${ }^{7}$ (All proofs are presented in the Appendix).

Proposition 1: For

$$
\theta \geq \bar{\theta}_{1}=\frac{1}{\rho P}\left(\frac{f \sigma}{I}\right)^{\frac{1}{\sigma-1}}
$$

firms choose the maximum effort $A$ without asking for a subsidy (i.e., firms have a hard budget constraint, HBC).

Proposition 2 (Segal, 1998): For a firm with $\bar{\theta}_{A} \leq \theta<\bar{\theta}_{1}$,

i) If $\pi(\theta, A)<s$, the firm chooses the cut-off level of effort $a^{0}(<A)$, receives the subsidy $S$ and produces (Moral hazard SBC);

ii) If $\pi(\theta, A)>s$, the firm chooses maximum effort $A$, receives no subsidy and produces (HBC);

iii) If $\pi(\theta, A)=s$, the result would be either i) or ii).

In Proposition 2, some firms with productivity $\theta \in\left[\bar{\theta}_{A}, \bar{\theta}_{1}\right)$ do not apply maximum effort in order to obtain the subsidy from the government, implying the existence of moral hazard.

Proposition 3: For a firm with $\theta<\bar{\theta}_{A}$,

i) If $\pi(\theta, A)+s \geq 0$, the firm chooses maximum effort $A$, receives a subsidy of $s$, and produces (Non-moral hazard SBC).

ii) If $\pi(\theta, A)+s<0$, the firm exits the market (HBC).

\footnotetext{
${ }^{7}$ As noted earlier. because we assume that relatively few firms are eligible for a subsidy, we are using the same price index as in the case without government subsidies calculated in expression (13) and, in particular, this price index integrates over all firms with productivites starting from $\bar{\theta}_{A}$ rather than from the threshold for the firms that are eligible for a subsidy. As shown below, this latter threshold, $\bar{\theta}_{N}$, is lower than $\bar{\theta}_{A}$, implying that strictly speaking the price index should have been $P=\left(N_{S} \int_{\bar{\theta}_{N}}^{\bar{\theta}_{A}}\left(\frac{1}{\rho \theta A}\right)^{1-\sigma} \gamma \theta^{-\gamma-1} d \theta+N \int_{\bar{\theta}_{A}}^{\infty}\left(\frac{1}{\rho \theta A}\right)^{1-\sigma} \gamma \theta^{-\gamma-1} d \theta\right)^{\frac{1}{1-\sigma}}$.
} 
In Proposition 3 some firms with $\theta<\bar{\theta}_{A}$ exert maximum effort $A$ and receive the subsidy to keep operating. So there is SBC, but no moral hazard is present.

\subsection{Cut-off Levels of Productivity for Moral Hazard SBC and Non-moral Hazard SBC}

Proposition 2 implies the existence of a cut-off level of productivity that determines whether a firm has moral hazard SBC or HBC. For $\theta \in\left[\bar{\theta}_{A}, \bar{\theta}_{1}\right)$ there exists $\bar{\theta}_{M}$ such that if a firm's productivity is greater than $\bar{\theta}_{M}$, then it will have HBC. Otherwise, a firm will have moral hazard SBC. This cut-off level is found from the condition $\left(\bar{\theta}_{M}, A\right)=\pi\left(\bar{\theta}_{M}, a^{0}\right)+s=s$ :

$$
\bar{\theta}_{M}=\frac{1}{\rho A P}\left[\frac{\sigma(f+s)}{I}\right]^{\frac{1}{\sigma-1}}
$$

Similarly, Proposition 3 implicitly defines the cut-off level of productivity $\bar{\theta}_{N}$ that determines whether the firm has non-moral hazard SBC or HBC (exit the market). This cut-off level is such that $\pi\left(\bar{\theta}_{N}, A\right)+s=0$ :

$$
\bar{\theta}_{N}=\frac{1}{\rho A P}\left[\frac{\sigma(f-s)}{I}\right]^{\frac{1}{\sigma-1}}
$$

Since we assume that $f>s, \bar{\theta}_{N}$ is greater than zero. If a firm's productivity is within $\left(\bar{\theta}_{N}, \bar{\theta}_{A},\right)$, it will have non-moral hazard SBC. If $\theta \leq \bar{\theta}_{N}$, a firm will have $\mathrm{HBC}$ and exit the market.

Some may argue that SBC usually characterizes large ("too big to fail") firms while in our model with uniform fixed costs, only firms with medium-level productivity and, therefore, medium-level output are subject to SBC. Note, however, that both productivity and output level of firms that choose to remain in business depends on their fixed costs. As is clear from (12) and (16), if we assume that SBC firms have significantly greater fixed costs than do non-SBC- 
eligible firms, then firms subject to SBC can be of arbitrarily large size measured by their output. Moreover, the fact that SBC firms have relatively high fixed costs accords nicely with common perception (e.g., large formerly state-owned firms in the economies in transition or Chaebols in Korea prior to the financial crisis of 1997-1998). At the same time, the assumption that SBCeligible firms have higher fixed costs than other firms does not affect any of our results either above or below. The same is true with respect to the model with trade, i.e., our results are not altered if SBC firms have higher export-related fixed costs than other firms.

So far we have defined four threshold levels of productivity, $\bar{\theta}_{1}, \bar{\theta}_{A}, \bar{\theta}_{M}$, and $\bar{\theta}_{N}$ in Eqs. (15), (12), (16), and (17), respectively. Because $0<s<f$, the ordering of the these cut-off levels is $0<\bar{\theta}_{N}<\bar{\theta}_{A}<\bar{\theta}_{M}<\bar{\theta}_{1} .{ }^{8}$. In sum, if the government is willing to provide a subsidy to some firms, the least productive of SBC-eligible firms with $\theta<\bar{\theta}_{N}$ will have $\mathrm{HBC}$ and choose not to produce, firms with $\bar{\theta}_{N} \leq \theta<\bar{\theta}_{A}$ will have non-moral hazard SBC, intermediate productivity firms with $\bar{\theta}_{A} \leq \theta<\bar{\theta}_{M}$ will have moral hazard SBC, and finally, most productive firms with $\theta \geq \bar{\theta}_{M}$ will have HBC.

\section{The Model with Trade}

Let the economy examined above engage in trade with another (foreign) economy that has the same parameters as the domestic economy except there is no SBC in the foreign country and that the home government does not subsidize foreign firms. When the home country opens its economy, some domestic firms start exporting to the foreign market and goods produced by

\footnotetext{
${ }^{8}$ To obtain $\bar{\theta}_{1}>\bar{\theta}_{M}$, we need to show that $s<f\left(A^{\sigma-1}-1\right)$. Since $\left(A^{\sigma-1}-1\right)>1$ as both $A$ and $\sigma$ are greater than 1 , and $s<f$, we obtain $s<f\left(A^{\sigma-1}-1\right)$.
} 
some foreign firms are imported. We describe the economy with trade below. As under autarky, domestic firms that are not eligible for government subsidies always apply maximum effort $A$. Firms that are potentially eligible for a subsidy might have an incentive to apply effort $a<A$ and play essentially the same game with the government as they do under autarky. The only

difference is that at Stage 4, firms make decisions not only about production, but also about whether to export.

\subsection{Production in the Domestic Market}

The equilibrium price, $p_{d}$, quantity, $q_{d}$, and profit, $\pi_{\mathrm{d}}$, in the domestic market given the aggregate price index in the open economy, $P_{T}$, and consumer income $I_{T}$ are as follows:

$$
\begin{gathered}
p_{d}(\theta, a)=\frac{1}{\rho \theta a} \\
q_{d}(\theta, a)=I_{T} P_{T}^{\sigma-1}(\rho \theta a)^{\sigma} \\
\pi_{d}(\theta, a)=\frac{I_{T}\left(\rho \theta a P_{T}\right)^{\sigma-1}}{\sigma}-f
\end{gathered}
$$

From $\pi_{d}(\theta, A)=0$, the cut-off level of productivity with maximum effort $A$ in the domestic market is:

$$
\bar{\theta}_{d A}=\frac{1}{\rho A P_{T}}\left(\frac{f \sigma}{I_{T}}\right)^{\frac{1}{\sigma-1}}
$$

When a firm makes no effort, that is, $a=1$, the cut-off level of productivity will be:

$$
\bar{\theta}_{d 1}=\frac{1}{\rho P_{T}}\left(\frac{f \sigma}{I_{T}}\right)^{\frac{1}{\sigma-1}}
$$


For firms with $\bar{\theta}_{d A} \leq \theta<\bar{\theta}_{d 1}$, their profits can be positive or negative, depending on their efforts, that is, $\pi_{\mathrm{d}}(\theta, 1)<0 \leq \pi_{\mathrm{d}}(\theta, \mathrm{A})$. Hence there exists the cut-off level of effort $a_{d}^{0}$ which satisfies $\pi_{d}\left(\theta, a_{d}^{0}\right)=0$ as follows:

$$
a_{d}^{0}(\theta)=\frac{1}{\rho \theta P_{T}}\left(\frac{f \sigma}{I_{T}}\right)^{\frac{1}{\sigma-1}}
$$

For firms with $\bar{\theta}_{d A} \leq \theta<\bar{\theta}_{d 1}$ we obtain the cut-off level of productivity for moral hazard SBC, $\bar{\theta}_{d M}$, in the domestic market such that $\pi_{d}\left(\bar{\theta}_{d M}, A\right)=\pi_{d}\left(\bar{\theta}_{d M}, a_{d}^{0}\right)+s=s$ :

$$
\bar{\theta}_{d M}=\frac{1}{\rho A P_{T}}\left[\frac{\sigma(f+s)}{I_{T}}\right]^{\frac{1}{\sigma-1}}
$$

For firms with $\theta<\bar{\theta}_{d A}$ there exists the cut-off level of productivity for non-moral hazard SBC such that $\pi_{d}\left(\bar{\theta}_{d N}, A\right)+s=0$ :

$$
\bar{\theta}_{d N}=\frac{1}{\rho A P_{T}}\left[\frac{\sigma(f-s)}{I_{T}}\right]^{\frac{1}{\sigma-1}}
$$

\subsection{Exports}

As in Melitz (2003), we define two new parameters related to trade costs. First, let $\tau>1$ be a per-unit iceberg costs for exporting such as transportation costs and tariffs. That is, exporters have to produce $\tau$ units of the good to sell one unit in the foreign market. In addition, let $f_{x}$ be the fixed cost of exporting. We assume that $f_{x}>f$. With these two types of trade frictions the total cost of exporting quantity $q_{x}, T C_{x}$ is:

$$
T C_{x}(\theta)=f_{x}+\left(\frac{\tau}{\theta a}\right) q_{x} .
$$


The firm's objective function in the foreign market is:

$$
\begin{gathered}
\max _{p_{x}} \pi_{x}(\theta, a)=p_{x} q_{x}-\frac{\tau}{\theta a} q_{x}-f_{x} \\
\text { subject to } \pi_{x}(\cdot) \geq 0
\end{gathered}
$$

From Eq. (27) we obtain the equilibrium price, quantity and profit for exporting as follows:

$$
\begin{gathered}
p_{x}(\theta, a)=\frac{\tau}{\rho \theta a} \\
q_{x}(\theta, a)=I_{T} P_{T}^{\sigma-1}\left(\frac{\rho \theta a}{\tau}\right)^{\sigma} \\
\pi_{x}(\theta, a)=\frac{I_{T}}{\sigma}\left(\frac{\rho \theta a P_{T}}{\tau}\right)^{\sigma-1}-f_{x}
\end{gathered}
$$

From the condition $\pi_{x}(\theta, a)=0$ the cut-off level of productivity for exporting with maximum effort $A$ is:

$$
\bar{\theta}_{x A}=\frac{\tau}{\rho A P_{T}}\left(\frac{f_{x} \sigma}{I_{T}}\right)^{\frac{1}{\sigma-1}}
$$

With no effort the cut-off level of productivity for exporting will be $\bar{\theta}_{x 1}=\frac{\tau}{\rho P_{T}}\left(\frac{f_{x} \sigma}{I_{T}}\right)^{\frac{1}{\sigma-1}}$, and $\bar{\theta}_{x 1}>\bar{\theta}_{x A}$ as $A>1$. Hence a firm with $\theta \geq \bar{\theta}_{x 1}$ will export regardless of its effort, while a firm with $\bar{\theta}_{x A} \leq \theta<\bar{\theta}_{x 1}$ will be a potential exporter depending on its effort. A firm with $\theta<\bar{\theta}_{x A}$ never exports. Finally, since foreign firms have the same productivity thresholds for export to the home country, the aggregate price index in the open economy is: 


$$
\begin{aligned}
& P_{T}=\left(N \int_{\bar{\theta}_{d A}}^{\infty} p_{d}(\theta, A)^{1-\sigma} d F(\theta)+N \int_{\bar{\theta}_{x A}}^{\infty} p_{x}(\theta, A)^{1-\sigma} d F(\theta)\right)^{\frac{1}{1-\sigma}} \\
& =\left(N \int_{\bar{\theta}_{d A}}^{\infty}\left(\frac{1}{\rho \theta A}\right)^{1-\sigma} \gamma \theta^{-\gamma-1} d \theta+N \int_{\bar{\theta}_{x A}}^{\infty}\left(\frac{\tau}{\rho \theta A}\right)^{1-\sigma} \gamma \theta^{-\gamma-1} d \theta\right)^{\frac{1}{1-\sigma}} \\
& \Rightarrow P_{T}=P_{T}\left(\bar{\theta}_{d A}, \bar{\theta}_{x A}\right)=\left(\frac{\gamma-(\sigma-1)}{\left(\frac{(\sigma-1) A}{\sigma}\right)^{\sigma-1} \gamma N\left[\left(\bar{\theta}_{d A}\right)^{\sigma-1-\gamma}+\left(\frac{1}{\tau}\right)^{\sigma-1}\left(\bar{\theta}_{x A}\right)^{\sigma-1-\gamma}\right]}\right)^{\frac{1}{\sigma-1}} \\
& \Rightarrow P_{T}=\left(\frac{\lambda}{\gamma(\rho A)^{\gamma}\left(\frac{\sigma}{I_{T}}\right)^{\beta}\left(f^{\beta}+\tau^{-\gamma} f_{x}^{\beta}\right)}\right)^{\frac{1}{\gamma}}
\end{aligned}
$$

where, as before, $\beta=1-\frac{\gamma}{\sigma-1}, \lambda=\gamma-(\sigma-1)$, and the last equality is obtained by using (21) and (31). Given our assumption that $N_{s}$ is sufficiently small so that subsidy-eligible firms do not affect income within the economy, it is straightforward to show that $I_{T}=I=\frac{\gamma}{\gamma-\rho} L .{ }^{9}$ Therefore, since $\tau^{-\gamma} f_{x}^{\beta}>0$, the aggregate price index in the open economy is less than that under autarky, i.e., $P_{T}<P$.

To examine how SBC affects the firms' decision to export we need to determine the ordering among productivity thresholds: $\bar{\theta}_{d N}, \bar{\theta}_{d M}, \bar{\theta}_{d A}, \bar{\theta}_{d 1}, \bar{\theta}_{x A}$, and $\bar{\theta}_{x 1}$. First, the productivity threshold beyond which firms applying maximum effort would export is greater than the threshold for simply producing in a non-SBC economy, i.e., $\bar{\theta}_{x A}>\bar{\theta}_{d A}$ as $\tau>1$ and $f_{x}>f$ in Eq. (21) and

\footnotetext{
${ }^{9}$ This result was first noted by Eaton and Kortum (2005) and is also proven in, for example, di Giovanni and Levchenko (2009). As noted earlier, for all of our results to hold we need to assume only that $N_{s}$ is sufficiently small so that it does not significantly affect the difference between $I$ and $I_{T}$ rather than each of the income values separately.
} 
Eq. (31). Second, the cut-off level of productivity for exporting without any effort, $\bar{\theta}_{x 1}$, has the highest value among these productivity thresholds. Third, the cut-off level of productivity for non-moral hazard SBC, $\bar{\theta}_{d N}$, has the lowest value among these productivity thresholds. Fourth, the upper productivity threshold for moral hazard SBC is greater than the minimum productivity threshold for choosing to produce in a non-SBC economy, i.e., $\bar{\theta}_{d M}>\bar{\theta}_{d A}$, because $s>0$. Fifth, the threshold for domestic production with no effort, $\bar{\theta}_{d 1}$, is greater than the cut-off level of productivity for moral hazard SBC, $\bar{\theta}_{d M}$, i.e., $\bar{\theta}_{d 1}>\bar{\theta}_{d M}$ because $s<f<f\left(A^{\sigma-1}-1\right)$.

As the following Lemma indicates, the rankings between $\bar{\theta}_{d M}$ and $\bar{\theta}_{x A}$, and between $\bar{\theta}_{d 1}$ and $\bar{\theta}_{x A}$, depend on the level of trade costs, $\mathrm{f}_{\mathrm{x}}$ and/or $\tau$.

Lemma: Denote an index of combined trade costs by $R, R \equiv \tau f_{x}^{1 /(\sigma-1)}$. Then,

(i) if $R>f^{1 /(\sigma-1)} A$, then $\bar{\theta}_{d N}<\bar{\theta}_{d A}<\bar{\theta}_{d M}<\bar{\theta}_{d 1}<\bar{\theta}_{x A}<\bar{\theta}_{x 1}$;

(ii) if $(f+s)^{1 /(\sigma-1)}<R<f^{1 /(\sigma-1)} A$, then $\bar{\theta}_{d N}<\bar{\theta}_{d A}<\bar{\theta}_{d M}<\bar{\theta}_{x A}<\bar{\theta}_{d 1}<\bar{\theta}_{x 1}{ }^{10}$;

(iii) if $R<(f+s)^{1 /(\sigma-1)}$, then $\bar{\theta}_{d N}<\bar{\theta}_{d A}<\bar{\theta}_{x A}<\bar{\theta}_{d M}<\bar{\theta}_{d 1}<\bar{\theta}_{x 1}$.

Proof. Straightforward.

As the Lemma and the discussion before it indicate, to the extent that the thresholds in the Lemma have counterparts in the autarky economy, the rankings and their implications for SBC do not change with the introduction of trade. More interestingly, the productivity threshold, $\bar{\theta}_{x A}$, beyond which firms would begin to export in an economy without SBC may be above or below the moral hazard SBC threshold, $\bar{\theta}_{d M}$, depending on the size of trade costs. As the following

$\overline{{ }^{10} \text { Note that } f^{1 /(\sigma-1)} A>(f+s)^{1 /(\sigma-1)}}$ because $s<\left(A^{\sigma-1}-1\right) f$ by assumption. 
Proposition shows, $\bar{\theta}_{x A}<\bar{\theta}_{d M}$ implies that some firms that would have exported in a non-SBC economy do not do so under SBC.

Proposition 4: Given Pareto distribution of firm productivities and a constant subsidy, $s$,

(i) if $R>(f+s)^{1 /(\sigma-1)}$, the presence of SBC has no effect on the firm's decision to export;

(ii) if $R<(f+s)^{1 /(\sigma-1)}$, the presence of SBC reduces the number of exporting firms. $\begin{array}{llll}\text { Specifically, } & \text { only firms with } & \text { productivities }\end{array}$

$$
\theta>\bar{\theta}_{x M}=\frac{1}{\rho A P_{T}}\left(\frac{\sigma\left(s+f+f_{x}\right)}{I_{T}} \cdot \frac{\tau^{\sigma-1}}{\tau^{\sigma-1}+1}\right)^{\frac{1}{\sigma-1}}>\bar{\theta}_{x A}
$$

will apply maximum effort and export;

(iii) in the presence of international trade, fewer firms have moral hazard SBC than under autarky.

We can now summarize the behavior of potentially SBC firms in the low trade cost environment as a function of firm productivity $\theta$ :

(i) $\theta \leq \bar{\theta}_{d N}$ : The least productive firms exit the market;

(ii) $\bar{\theta}_{d N} \leq \theta \leq \bar{\theta}_{d A}$ : The low productivity firms have non-moral hazard SBC and serve only the domestic market;

(iii) $\bar{\theta}_{d A} \leq \theta \leq \bar{\theta}_{x M}$ : The firms with intermediate productivity have moral hazard SBC and serve only the domestic market;

(iv) $\bar{\theta}_{x M} \leq \theta$ : The most productive firms have $\mathrm{HBC}$ and serve both the domestic and the foreign market. 


\subsection{Comparative statics}

In this section we examine the impact of changes in trade costs, both $f_{x}$ and $\tau$, on the amount of subsidies paid by the government and the extent of SBC-induced inefficiency due to lower than maximum effort applied by the potentially SBC firms. Recall that there are $N_{s}$ firms eligible for a subsidy. Of these, according to the Lemma and to Proposition 4, the firms that apply low effort and actually receive a subsidy have productivities in the $\left(\bar{\theta}_{d N}, \bar{\theta}_{d M}\right)$ interval if $R>$ $(f+s)^{1 /(\sigma-1)}$ and in the $\left(\bar{\theta}_{d N}, \bar{\theta}_{x M}\right)$ interval if $R<(f+s)^{1 /(\sigma-1)}$. Therefore, the total subsidy, S, provided by the government is given by the following two expressions:

$$
\begin{aligned}
& S=s N_{S}\left(F\left(\bar{\theta}_{d M}\right)-F\left(\bar{\theta}_{d N}\right)\right) \text { if } R>(f+s)^{1 /(\sigma-1)} \\
& S=s N_{S}\left(F\left(\bar{\theta}_{x M}\right)-F\left(\bar{\theta}_{d N}\right)\right) \text { if } R<(f+s)^{1 /(\sigma-1)}
\end{aligned}
$$

The SBC-induced inefficiency due to lower than socially efficient effort (i.e., effort lower than $A$ ) applied by the firms can be measured by: ${ }^{11}$

$$
\Omega=N_{s} \int_{\bar{\theta}_{d A}}^{\bar{\theta}_{d M}}\left(\pi_{d}(\theta, A)-\pi_{d}(\theta, a)\right) d F(\theta) \text { if } R>(f+s)^{1 /(\sigma-1)}
$$

and

$$
\begin{array}{r}
\Omega=N_{s}\left(\int_{\bar{\theta}_{d A}}^{\bar{\theta}_{x A}}\left(\pi_{d}(\theta, A)-\pi_{d}(\theta, a)\right) d F(\theta)+\int_{\bar{\theta}_{x A}}^{\bar{\theta}_{x M}}\left(\pi_{x}(\theta, A)-\pi_{d}(\theta, a)\right) d F(\theta)\right) \\
\text { if } R<(f+s)^{1 /(\sigma-1)}
\end{array}
$$

\footnotetext{
${ }^{11}$ Strictly speaking, these expressions presumably overestimate welfare loss somewhat, because on average additional output produced with extra effort would be less valuable than marginal output. This consideration, however, does not affect the comparative statics below.
} 
where $a=\frac{1}{\rho \theta P_{T}}\left(\frac{f \sigma}{I_{T}}\right)^{\frac{1}{\sigma-1}}$ and the respective profit functions are defined in (20) and (30). In addition, SBC generates inefficiencies by keeping afloat firms that applied maximum effort but would have gone out of business if not for the subsidies. This inefficiency can be measured by the following expression:

$$
\Omega_{0}=N_{s} \int_{\bar{\theta}_{d N}}^{\bar{\theta}_{d A}}\left(s-\pi_{d}(\theta, A)\right) d F(\theta)
$$

By differentiating expressions (34)-(38) with respect to $\tau$ and $f_{x}$, we obtain the following Proposition.

Proposition 5. Trade liberalization reflected in the decline of trade costs $\tau$ and $f_{x}$ reduces both the total amount of subsidy paid by the government to eligible firms and the SBC-induced inefficiencies as measured by (36)-(38).

This result is obtained under the assumption of an exogenously fixed amount of subsidy to an individual firm. One may argue that as trade liberalization results in smaller aggregate subsidy and, therefore, creates some "leftover" government funds, firms may try to lobby for increasing the size of individual subsidy or firms that were not previously eligible for a subsidy might try to obtain it. Whether such lobbying is likely to succeed depends on the trade-induced changes in social costs and benefits of subsidies and the opportunity cost of subsidies to the government, among other factors. For example, trade liberalization might result in government reallocating some funds for export promotion, increasing the opportunity cost of supporting SBC firms. In this case, one distortion might reduce incentives for another distortion similarly to the 
mechanism analyzed in Qian and Roland (1998). These issues are beyond the scope of the present paper and may present a fruitful direction for future research.

\section{Conclusion}

In this paper we analyze the interaction of the soft budget constraint (SBC) and international trade liberalization by placing Segal's (1998) SBC model within Melitz's (2003) framework of international trade with heterogeneous monopolistically competitive firms. We first examine the relationship between SBC and firm productivity under autarky. The least productive firms eligible for a subsidy have HBC and exit the market, the next tier of firms have non-moral hazard SBC (i.e., they apply maximum effort and receive a subsidy), the intermediate productivity firms have moral hazard SBC, and the most productive firms have HBC. Therefore, among potentially SBC firms only the intermediate productivity firms apply suboptimal effort in order to obtain a subsidy from government. The inefficiency generated by SBC, however, includes both the profits lost by firms subject to moral hazard SBC and the waste of resources by relatively low productivity $\mathrm{HBC}$ firms that are kept afloat by the subsidies. Note that by assuming that potentially SBC firms have higher fixed costs than other firms, the above results would imply that moral hazard SBC affects large firms as measured by their output.

The possibility of international trade adds another sort of inefficiency. Some firms that would have become exporters in the absence of SBC choose to apply low effort and not export in order to extract a subsidy from the government. This effect takes place when trade costs are sufficiently low. Overall, however, trade liberalization reduces inefficiencies generated by SBC. The total amount of the subsidy and, therefore, the number of firms subject to moral hazard SBC decrease as trade costs decline. More important, aggregate effort level increases and aggregate 
profits lost due to SBC-induced sub-optimal effort decline as trade costs decrease. Also, trade liberalization reduces social loss induced by subsidies that keep afloat firms that would have exited the market if no subsidies were available.

\section{References}

Alexeev, Michael and Sunghwan Kim. (2004), “Lenders’ Reputation and the Soft Budget Constraint," Economics Letters, 84:69-73.

Chaney, Thomas, 2005. "Liquidity constrained exporters," Working Paper, University of Chicago.

Dewatripont, Mathias and Eric Maskin. (1995), "Credit and Efficiency in Centralized and Decentralized Economies," Review of Economic Studies, 62:4, pp. 541-55.

di Giovanni, Julian, and Andrei Levchenko, 2009. "Firm Entry, Trade, and Welfare in Zipf's World," RSIE Discussion Paper 591.

Do, Quy-Toan, and Andrei Levchenko, 2009. "Trade, Inequality, and the Political Economy of Institutions," Journal of Economic Theory, 144(4):1489-1520.

Eaton, Jonathan and Samuel Kortum, 2005. Technology in the Global Economy: A Framework for Quantitative Analysis, book manuscript, New York University and University of Chicago.

Kornai, Janos. (1979), "Resource-Constrained Versus Demand-Constrained Systems," Econometica,47:4, pp.801-19.

Kornai, Janos. (1980), Economics of Shortage, Amsterdam: North-Holland. 
Kornai, Janos, Eric Maskin and Gerard Roland. (2003), "Understanding the Soft Budget Constraint," Journal of Economic Literature 41:4, pp.1095-1136.

Maskin, Eric and Chenggang Xu. (2001), "Soft Budget Constraint Theories: From Centralization to the Market," Economics of Transition 9:1, pp.1-27.

Melitz, Marc. (2003), “The Impact of Trade on Intra-Industry Reallocations and Aggregate Industry Productivity,” Econometrica 71:6, pp. 1695-1725.

Qian, Yingyi, and Gérard Roland (1998), "Federalism and the Soft Budget Constraint," American Economic Review, 88,5:1143-61.

Segal, Ilya. (1998), "Monopoly and Soft Budge Constraint," RAND Journal of Economics 29:3, pp.569-609. 


\section{Appendix}

Proposition 1: For

$$
\theta \geq \bar{\theta}_{1}=\frac{1}{\rho P}\left(\frac{f \sigma}{I}\right)^{\frac{1}{\sigma-1}}
$$

firms choose the maximum effort $A$ without asking for any subsidy (HBC).

Proof of Proposition 1 : First, note that from (12) the cut-off level of productivity for a firm that does not apply any effort is $\bar{\theta}_{1}=\frac{1}{\rho P}\left(\frac{f \sigma}{I}\right)^{\frac{1}{\sigma-1}}$ and given that $A>1, \bar{\theta}_{1}>\bar{\theta}_{A}$. Hence, firms with $\theta \geq \bar{\theta}_{1}$ will always obtain positive profits and, therefore, operate regardless of effort, i.e., for these firms, $0 \leq \pi(\theta, 1)<\pi(\theta, A)$.

By backward induction, in Stage 4 , the firm with $\theta \geq \bar{\theta}_{1}$ will produce even without $s$. Hence, the firm's threat not to produce without $s$ is not credible. Therefore, in Stage 3, the government will reject the request to provide a subsidy. In Stage 2, the firm knows that the government will not accept its request; so it will not ask for a subsidy and will choose effort $A$ in Stage 1 to maximize its profit without the subsidy. Q.E.D.

Proposition 2 (Segal, 1998): For a firm with $\bar{\theta}_{A} \leq \theta<\bar{\theta}_{1}$,

i) If $\pi(\theta, A)<s$, the firm chooses the cut-off level of effort $a^{0}(<A)$, receives the subsidy $s$ and produces (Moral hazard SBC);

ii) If $\pi(\theta, A)>s$, the firm chooses maximum effort $A$, receives no subsidy and produces (HBC).

iii) If $\pi(\theta, A)=s$, the result would be either i) or ii). 
Proof of Proposition 2: (i) By backward induction, if in Stage 4, $\pi(\theta, a) \leq 0$, the firm will not produce without a subsidy. Therefore, in Stage 3, the government will accept the firm's request to provide a subsidy if $\pi(\theta, a) \leq 0$. If, however, in Stage $4, \pi(\theta, a)>0$, the firm would produce even without a subsidy and in Stage 3 the government would not accept the firm's request for a subsidy. Knowing this, in Stage 2, the firm will ask for $s$ if $\pi(\theta, a) \leq 0$. In Stage 1, the firm knows that by choosing $a \leq a^{0}$ it can obtain benefit $\pi(\theta, a)+s \geq s$ and by choosing $a=A$ it would obtain profit $(\theta, A)<s$. Therefore, to maximize its profit, the firm will choose $a^{0}$ to obtain $\left(\theta, a^{0}\right)+s=s$. Effort level $a^{0}$ exists because without a subsidy, and given $\bar{\theta}_{A} \leq \theta<\bar{\theta}_{1}$, the firm's profit can be negative or non-negative depending on its effort, i.e., $\pi(\theta, 1)<0 \leq$ $\pi(\theta, A)$. Given that $\pi(\theta, a)$ is continuous in $a$, a cut-off level of effort $a^{0}$ exists and satisfies $\pi\left(\theta, a^{0}\right)=0$ :

$$
\pi\left(\theta, a^{0}\right)=\frac{I(\rho \theta a P)^{\sigma-1}}{\sigma}-f=0,
$$

$\Rightarrow a^{0}(\theta)=\frac{1}{\rho \theta P}\left(\frac{f \sigma}{I}\right)^{\frac{1}{\sigma-1}}=\frac{1}{\theta} \bar{\theta}_{1}$.(ii) Using reasoning similar to that in (i), we find that the firm would choose $a=A$ in order to obtain profit $\pi(\theta, A)>s$.

(iii) Here, the firm is indifferent between applying effort $a^{0}$ and receiving $s$ and applying effort $A$ and receiving no subsidy. Therefore, either is possible. Q.E.D.

Proposition 3: For a firm with $\theta<\bar{\theta}_{A}$,

i) If $\pi(\theta, A)+s \geq 0$, the firm chooses maximum effort $A$, receives a subsidy of $s$, and produces (Non-moral hazard SBC).

ii) If $\pi(\theta, A)+s<0$, the firm exits the market (HBC). 
Proof of Proposition 3 : (i) By backward induction, in Stage 4, the firm's threat not to produce is credible because $\pi(\theta, a)<0, \forall a$. In Stage 3, the government will accept the firm's request for subsidy. Knowing this, in Stage 2, the firm will ask for a subsidy. In Stage 1, the firm's profit is $\pi(\theta, a)+s \leq \pi(\theta, A)+s$. Hence, the firm will choose $A$ to maximize its profit.

(ii) If $\pi(\theta, A)+s<0$, the firm's payoff with or without a subsidy is negative and the firm will exit. Q.E.D.

Proposition 4: Given Pareto distribution of firm productivities and a constant subsidy, $s$,

(i) if $R>(f+s)^{1 /(\sigma-1)}$, the presence of SBC has no effect on the firm's decision to export;

(ii) if $R<(f+s)^{1 /(\sigma-1)}$, the presence of SBC reduces the number of exporting firms. $\begin{array}{llll}\text { Specifically, } & \text { only firms with } & \text { productivities }\end{array}$

$$
\theta>\bar{\theta}_{x M}=\frac{1}{\rho A P_{T}}\left(\frac{\sigma\left(s+f+f_{x}\right)}{I} \cdot \frac{\tau^{\sigma-1}}{\tau^{\sigma-1}+1}\right)^{\frac{1}{\sigma-1}}>\bar{\theta}_{x A}
$$

will apply maximum effort and export;

(iii) in the presence of international trade, fewer firms have moral hazard SBC than under autarky.

Proof of Proposition 4:

(i) follows directly from the fact that for $R>(f+s)^{1 /(\sigma-1)}, \bar{\theta}_{d M}<\bar{\theta}_{x A}$, (see the Lemma).

(ii) If $R<(f+s)^{1 /(\sigma-1)}$, then $\bar{\theta}_{x A}<\bar{\theta}_{d M}$ and, therefore, firms with productivities $\bar{\theta}_{x A}<\theta<\bar{\theta}_{d M}$ that are eligible for a subsidy would choose to apply effort $a_{d}^{0}<$ 
$A$, such that $\pi_{d}\left(\theta, a_{d}^{0}\right)=0$, receive the subsidy and produce only in the domestic market (moral hazard SBC). Threshold (33) in the Proposition is obtained from solving the equation $\pi_{T}\left(\bar{\theta}_{x M}, A\right)=\pi_{d}\left(\bar{\theta}_{x M}, A\right)+\pi_{x}\left(\bar{\theta}_{x M}, A\right)=s$ with respect to $\bar{\theta}_{x M}$. In the absence of SBC, all firms with $\theta>\bar{\theta}_{x A}$ would have applied effort $A$ and produced both for the domestic market and for export.

(iii) Recall that the number of potentially SBC firms is denoted by $N_{s}$. Under autarky, the expected number of moral hazard SBC firms equals $N_{S}\left(F\left(\bar{\theta}_{M}\right)-F\left(\bar{\theta}_{A}\right)\right)$, where $F($.$) represents cumulative distribution function of SBC firms'$ productivities. With trade, the expected number of moral hazard SBC firms is given by $N_{s}\left(\operatorname{Min}\left\{F\left(\bar{\theta}_{d M}\right), F\left(\bar{\theta}_{x M}\right)\right\}-F\left(\bar{\theta}_{d A}\right)\right)$. Suppose first that $\bar{\theta}_{d M} \leq \bar{\theta}_{x M}$. Given Pareto distribution of firm productivities and that thresholds $\bar{\theta}_{d M}$ and $\bar{\theta}_{M}$ and $\bar{\theta}_{d A}$ and $\bar{\theta}_{A}$ differ only by the inverse of the respective aggregate price indices, the sign of $F\left(\bar{\theta}_{d M}\right)-F\left(\bar{\theta}_{d A}\right)-\left(F\left(\bar{\theta}_{M}\right)-F\left(\bar{\theta}_{A}\right)\right)$ is the same, as the sign of $P_{T}^{\gamma}-P^{\gamma}<0$. If $\bar{\theta}_{d M}>\bar{\theta}_{x M}$, then $F\left(\bar{\theta}_{d M}\right)>F\left(\bar{\theta}_{x M}\right)$ implying that $F\left(\bar{\theta}_{x M}\right)-F\left(\bar{\theta}_{d A}\right)-\left(F\left(\bar{\theta}_{M}\right)-F\left(\bar{\theta}_{A}\right)\right)$ is also negative. Q.E.D.

Proposition 5. Trade liberalization reflected in the decline of trade costs $\tau$ and $f_{x}$ reduces both the total amount of subsidy paid by the government to eligible firms and the SBC-induced inefficiency.

Proof. First, note that the expression for $P_{T}$ in (32) implies that $\frac{\partial P_{T}}{\partial \tau}>0$ and $\frac{\partial P_{T}}{\partial f_{x}}>0$. Also, from (24), $\frac{\partial \bar{\theta}_{d M}}{\partial \tau}=\frac{-1}{\rho A P_{T}^{2}}\left[\frac{\sigma(f+s)}{I}\right]^{\frac{1}{\sigma-1}} \frac{\partial P_{T}}{\partial \tau}$ and from (25), $\frac{\partial \bar{\theta}_{d N}}{\partial \tau}=\frac{-1}{\rho A P_{T}^{2}}\left[\frac{\sigma(f-s)}{I}\right]^{\frac{1}{\sigma-1}} \frac{\partial P_{T}}{\partial \tau}$. (Note that under our assumptions, $I_{T}=I$ for all values of $\tau$ and $f_{x}$ and, therefore, $\frac{\partial I_{T}}{\partial \tau}=\frac{\partial I_{T}}{\partial f_{x}}=0$.) Similarly, 
$\frac{\partial \bar{\theta}_{d M}}{\partial f_{x}}=\frac{-1}{\rho A P_{T}^{2}}\left[\frac{\sigma(f+s)}{I}\right]^{\frac{1}{\sigma-1}} \frac{\partial P_{T}}{\partial f_{x}}$ and $\frac{\partial \bar{\theta}_{d N}}{\partial f_{x}}=\frac{-1}{\rho A P_{T}^{2}}\left[\frac{\sigma(f-s)}{I}\right]^{\frac{1}{\sigma-1}} \frac{\partial P_{T}}{\partial f_{x}}$. From these expressions and using (34), it is straightforward to show that for $R>(f+s)^{1 /(\sigma-1)}$, the signs of $\frac{\partial S}{\partial \tau}$ and $\frac{\partial S}{\partial f_{x}}$ are the same as the sign of $\left([f+s]^{\frac{-\gamma}{\sigma-1}}-[f-s]^{\frac{-\gamma}{\sigma-1}}\right)\left(-\frac{\partial P_{T}}{\partial z}\right)$, where $z$ stands either for $\tau$ or for $f_{x}$. Therefore, $\frac{\partial S}{\partial \tau}>0$ and $\frac{\partial S}{\partial f_{x}}>0$. If $R<(f+s)^{1 /(\sigma-1)}$, we need to replace $\bar{\theta}_{d M}$ with $\bar{\theta}_{x M}$ in the above calculations. Specifically, $\frac{\partial \bar{\theta}_{x M}}{\partial \tau}=\frac{\partial \bar{\theta}_{x M}}{\partial \tau}+\frac{\partial \bar{\theta}_{x M}}{\partial P_{T}} \frac{\partial P_{T}}{\partial \tau}>\frac{\partial \bar{\theta}_{x M}}{\partial P_{T}} \frac{\partial P_{T}}{\partial \tau}$ and $\frac{\partial \bar{\theta}_{x M}}{\partial f_{x}}=\frac{\partial \bar{\theta}_{x M}}{\partial f_{x}}+$ $\frac{\partial \bar{\theta}_{x M}}{\partial P_{T}} \frac{\partial P_{T}}{\partial f_{x}}>\frac{\partial \bar{\theta}_{x M}}{\partial P_{T}} \frac{\partial P_{T}}{\partial f_{x}}$. Therefore, the signs of $\frac{\partial S}{\partial \tau}$ and $\frac{\partial S}{\partial f_{x}}$ are the same as

$$
\left(\left[\left(f+f_{x}+s\right) \frac{\tau^{\sigma-1}}{\tau^{\sigma-1}+1}\right]^{\frac{-\gamma}{\sigma-1}}-[f-s]^{\frac{-\gamma}{\sigma-1}}\right)\left(-\frac{\partial P_{T}}{\partial z}\right)
$$

where $z$ again stands either for $\tau$ or for $f_{x}$. Note that expression $\frac{\tau^{\sigma-1}}{\tau^{\sigma-1}+1}$ reaches its minimum feasible value of $1 / 2$ when $\tau=1$. Also, because $f_{x}>f$, expression (39) is greater than $([(2 f+$ $\left.s) / 2]^{\frac{-\gamma}{\sigma-1}}-[f-s]^{\frac{-\gamma}{\sigma-1}}\right)\left(-\frac{\partial P_{T}}{\partial z}\right)>0$. That is, the total amount of subsidy paid by the government diminishes as trade costs decline.

We now turn to comparative statics of the aggregate inefficiency induced SBC. Consider first $\frac{\partial \Omega}{\partial z}$ for $R>(f+s)^{1 /(\sigma-1)}$. Because $\frac{\partial P_{T}}{\partial z}>0$, the sign of this derivative is the same as the sign of the derivative of the integral from (36). This integral is: 


$$
\begin{gathered}
\int_{\bar{\theta}_{d A}}^{\bar{\theta}_{d M}}\left(\pi_{d}(\theta, A)-\pi_{d}(\theta, a)\right) d F(\theta) \\
=\frac{I}{\sigma \beta}\left(\rho P_{T} A\right)^{\gamma}\left(H_{1} \beta\left(H_{2}^{\beta-1}-H_{1}^{\beta-1}\right)+(1-\beta)\left(H_{2}^{\beta}-H_{1}^{\beta}\right)\right),
\end{gathered}
$$

where $H_{1}=\frac{\sigma f}{I}, H_{2}=\frac{\sigma(f+s)}{I}$ and, as before, $\beta=1-\frac{\gamma}{\sigma-1}$. Its derivative with respect to $P_{T}$ is:

$$
\frac{\gamma I\left(\rho P_{T} A\right)^{\gamma}}{\sigma P_{T} \beta}\left(\beta H_{2}^{\beta-1} H_{1}+(1-\beta) H_{2}^{\beta}-H_{1}^{\beta}\right)
$$

Because $\beta<0$ the sign of (41) is opposite to the sign of the expression in the large parentheses. Using the expressions for $H_{1}, H_{2}$, and $\beta$, the expression in the parentheses can be rewritten as

$\left(\frac{\sigma}{I}\right)^{\beta}\left(\left(f+\frac{\gamma s}{\sigma-1}\right)(f+s)^{-\frac{\gamma}{\sigma-1}}-f^{1-\frac{\gamma}{\sigma-1}}\right)<\left(\frac{\sigma}{I}\right)^{\beta}\left(\left(f+\frac{\gamma f}{\sigma-1}\right)(f+f)^{-\frac{\gamma}{\sigma-1}}-f^{1-\frac{\gamma}{\sigma-1}}\right)=$ $\left(\frac{\sigma}{I}\right)^{\beta}\left(2^{\frac{-\gamma}{\sigma-1}}\left(1+\frac{\gamma}{\sigma-1}\right) f^{1-\frac{\gamma}{\sigma-1}}-f^{1-\frac{\gamma}{\sigma-1}}\right)=\left(\frac{\sigma f}{I}\right)^{\beta} 2^{\frac{-\gamma}{\sigma-1}}\left(1+\frac{\gamma}{\sigma-1}-2^{\frac{\gamma}{\sigma-1}}\right)$

The last parentheses in (42) are negative. This is because the expression in the parentheses is maximized at the lowest feasible value of $\frac{\gamma}{\sigma-1}>1$ and at the point where $\frac{\gamma}{\sigma-1}=1$ the parentheses are zero. Therefore, expression (41) is positive and given that $\frac{\partial P_{T}}{\partial z}>0$, we obtain that when $R>(f+s)^{1 /(\sigma-1)}$, efficiency increases as trade costs fall.

We now turn to the case of $R<(f+s)^{1 /(\sigma-1)}$. Denote

$$
\Omega_{1}=\int_{\bar{\theta}_{d A}}^{\bar{\theta}_{x A}}\left(\pi_{d}(\theta, A)-\pi_{d}(\theta, a)\right) d F(\theta) \text { and }
$$




$$
\Omega_{2}=\int_{\bar{\theta}_{x A}}^{\bar{\theta}_{x M}}\left(\pi_{x}(\theta, A)-\pi_{d}(\theta, a)\right) d F(\theta)
$$

(We do not include $N_{S}$ in these expressions because it is not going to affect the sign of the derivatives.) We will first show that $\frac{\partial \Omega_{1}}{\partial z}=\frac{\partial \Omega_{1}}{\partial z}+\frac{\partial \Omega_{1}}{\partial P_{T}} \frac{\partial P_{T}}{\partial z}>0$ and then we will show that $\frac{\partial \Omega_{2}}{\partial z}=\frac{\partial \Omega_{2}}{\partial z}+\frac{\partial \Omega_{2}}{\partial P_{T}} \frac{\partial P_{T}}{\partial z}>0$ (where, as before, $z$ stands either for $\tau$ or for $f_{x}$ ). Consider first $\frac{\partial \Omega_{1}}{\partial P_{T}}$. Similarly to (41), this derivative reduces to the following expression:

$$
\frac{\gamma I\left(\rho P_{T} A\right)^{\gamma}}{\sigma P_{T} \beta}\left(H_{1} \beta\left(\tau^{-\gamma} H_{3}^{\beta-1}-H_{1}^{\beta-1}\right)+(1-\beta)\left(\tau^{-\gamma} H_{3}^{\beta}-H_{1}^{\beta}\right)\right),
$$

where $H_{1}$ and $\beta$ were defined before and $H_{3}=\frac{\sigma f_{x}}{I}$. Again, the sign of (44) is opposite to the sign of the large parentheses. The latter can be written as

$$
\left(\frac{\sigma}{I}\right)^{\beta}\left(\tau^{-\gamma}\left(f \beta f_{x}^{\beta-1}+(1-\beta) f_{x}^{\beta}-f^{\beta} \tau^{\gamma}\right)\right)<\left(\frac{\sigma}{I}\right)^{\beta}\left(f \beta f_{x}^{\beta-1}+(1-\beta) f_{x}^{\beta}-f^{\beta} \tau^{\gamma}\right)
$$

The derivative of the latter expression with respect to $f_{x}$ is negative, implying that this expression reaches its maximum at the lowest feasible value of $f_{x}=f$. At this point the right hand side of (44) is zero, implying that for all $f_{x}>f$ the right hand side of (45) is negative and, therefore, $\frac{\partial \Omega_{1}}{\partial P_{T}}>0$. Consider next $\frac{\partial \Omega_{1}}{\partial \tau}$ :

$$
\frac{\partial \Omega_{1}}{\partial \tau}=\frac{\gamma I\left(\rho P_{T} A\right)^{\gamma}}{\sigma \tau^{1+\gamma}} H_{3}^{\beta}\left(\tau-H_{1} H_{3}^{-1}\right)>0
$$

It is similarly straightforward to show that $\frac{\partial \Omega_{1}}{\partial f_{x}}>0$. Therefore, $\frac{\partial \Omega_{1}}{\partial z}=\frac{\partial \Omega_{1}}{\partial z}+\frac{\partial \Omega_{1}}{\partial P_{T}} \frac{\partial P_{T}}{\partial z}>0$. 
Now we turn to $\frac{\partial \Omega_{2}}{\partial z}=\frac{\partial \Omega_{2}}{\partial z}+\frac{\partial \Omega_{2}}{\partial P_{T}} \frac{\partial P_{T}}{\partial z}$. Consider first $\frac{\partial \Omega_{2}}{\partial P_{T}}$. It can be simplified to

$$
\begin{gathered}
\frac{\partial \Omega_{2}}{\partial P_{T}}=\frac{\gamma I\left(\rho P_{T} A\right)^{\gamma}}{\sigma P_{T} \beta}\left(-\beta H_{3}\left(\tau^{-\gamma} H_{3}^{\beta-1}-H_{4}^{\beta-1}\right)-(1-\beta)\left(\tau^{-\gamma} H_{3}^{\beta}-H_{4}^{\beta} \tau^{-(\sigma-1)}\right)\right) \\
=\frac{\gamma I\left(\rho P_{T} A\right)^{\gamma}}{\sigma P_{T} \beta}\left(\beta H_{3} H_{4}^{\beta-1}-\tau^{-\gamma} H_{3}^{\beta}+(1-\beta) H_{4}^{\beta} \tau^{-(\sigma-1)}\right)
\end{gathered}
$$

where $H_{4}=\frac{\sigma\left(s+f+f_{x}\right)}{I} \cdot \frac{\tau^{\sigma-1}}{\tau^{\sigma-1}+1}$. Notice that $R=\tau f_{x}^{1 /(\sigma-1)}<(f+s)^{1 /(\sigma-1)}$ implies that $H_{4}>$ $\frac{\sigma\left(\tau^{\sigma-1} f_{x}+f_{x}\right)}{I} \cdot \frac{\tau^{\sigma-1}}{\tau^{\sigma-1}+1}=H_{3} \tau^{\sigma-1}$ or $\frac{H_{3}}{H_{4}}<\tau^{-(\sigma-1)}$. Denote the expression the last parentheses in (46) by $G=\beta H_{3} H_{4}^{\beta-1}-\tau^{-\gamma} H_{3}^{\beta}+(1-\beta) H_{4}^{\beta} \tau^{-(\sigma-1)}$. Notice that

$$
\frac{\partial G}{\partial H_{4}}=\beta(\beta-1) H_{4}^{\beta-1}\left(\frac{H_{3}}{H_{4}}-\tau^{-(\sigma-1)}\right)<0
$$

Therefore, if we replace $H_{4}$ in $G$ with $H_{3} \tau^{\sigma-1}$, the resulting expression will be greater than $G$ :

$$
G<\beta H_{3}\left(H_{3} \tau^{\sigma-1}\right)^{\beta-1}-\tau^{-\gamma} H_{3}^{\beta}+(1-\beta)\left(H_{3} \tau^{\sigma-1}\right)^{\beta} \tau^{-(\sigma-1)}=\tau^{-\gamma} H_{3}^{\beta}-\tau^{-\gamma} H_{3}^{\beta}=0 .
$$

This demonstrates that $G$ is negative and, therefore, (46) is positive. In order to show that $\frac{\partial \Omega_{1}}{\partial \tau}>0$, we will show that each term in $\frac{\partial \Omega_{2}}{\partial \tau}=\frac{\partial \Omega_{2}}{\partial \tau}+\frac{\partial \Omega_{2}}{\partial H_{4}} \frac{\partial H_{4}}{\partial \tau}$ is positive. First note:

$$
\frac{\partial \Omega_{2}}{\partial \tau}=\frac{\gamma I\left(\rho P_{T} A\right)^{\gamma}}{\sigma P_{T} \beta}\left(\beta \tau^{-\gamma} H_{3}^{\beta}-\beta H_{4}^{\beta} \tau^{-(\sigma-1)}\right)=\frac{\gamma I\left(\rho P_{T} A\right)^{\gamma}}{\sigma P_{T}}\left(\tau^{-\gamma} H_{3}^{\beta}-H_{4}^{\beta} \tau^{-(\sigma-1)}\right)>0
$$

Also, $\quad \frac{\partial \Omega_{2}}{\partial H_{4}}=\frac{(\beta-1) I\left(\rho P_{T} A\right)^{\gamma}}{\sigma H_{4}}\left(H_{3} H_{4}^{\beta-1}-H_{4}^{\beta} \tau^{-(\sigma-1)}\right)=\frac{(1-\beta) I\left(\rho P_{T} A\right)^{\gamma}}{\sigma H_{4}^{1-\beta}}\left(\tau^{-(\sigma-1)}-\frac{H_{3}}{H_{4}}\right)>0 \quad . \quad$ In addition, it is straightforward to show that $\frac{\partial H_{4}}{\partial \tau}>0$.Using similar approach, we can easily show 
that $\frac{\partial \Omega_{2}}{\partial f_{x}}>0$. The above arguments establish that both integrals in (43) and, therefore, expression (37) decline as trade costs decrease.

Finally, we need to demonstrate that $\frac{\partial \Omega_{0}}{\partial z}>0$, where $\Omega_{0}$ is given in (38). Note that $\Omega_{0}$ depends on $z$ only via $P_{T}$. Therefore, all we need to show is that $\frac{\partial \Omega_{0}}{\partial P_{T}}>0$. This can be done through already familiar steps. Denote $H_{5}=\frac{s \sigma}{I}$ and $H_{6}=\frac{(f-s) \sigma}{I}$, and note that $H_{6}<H_{1}$. Then,

$$
\begin{aligned}
\frac{\partial \Omega_{0}}{\partial P_{T}}=\frac{\gamma I\left(\rho P_{T} A\right)^{\gamma}}{\sigma P_{T} \beta} & \left(\beta H_{5} H_{6}^{\beta-1}-\beta H_{5} H_{1}^{\beta-1}-H_{1}^{\beta}+\beta H_{1} H_{6}^{\beta-1}+(1-\beta) H_{6}^{\beta}\right) \\
= & \frac{\gamma I\left(\rho P_{T} A\right)^{\gamma}}{\sigma P_{T} \beta}\left(\beta H_{5}\left(H_{6}^{\beta-1}-H_{1}^{\beta-1}\right)-H_{1}^{\beta}+\beta H_{1} H_{6}^{\beta-1}+(1-\beta) H_{6}^{\beta}\right)
\end{aligned}
$$

In the above expression, the fraction in front of the parentheses is negative. Also, the derivative of the expression in the parentheses with respect to $H_{1}$ is:

$$
-\beta^{2} H_{5} H_{1}^{\beta-2}+\beta H_{5} H_{1}^{\beta-2}-\beta\left(H_{1}^{\beta-1}-H_{6}^{\beta-1}\right)
$$

The first two terms in (49) are negative and the expression in the parentheses in the last term is negative, because $H_{1}>H_{6}$. Therefore, the derivative of (48) with respect to $H_{1}$ is negative, implying that its maximum value is reached at the minimum feasible value of $H_{1}$. Replacing $H_{1}$ in (48) with $H_{6}$, we obtain:

$$
\begin{gathered}
\beta H_{5}\left(H_{6}^{\beta-1}-H_{1}^{\beta-1}\right)-H_{1}^{\beta}+\beta H_{1} H_{6}^{\beta-1}+(1-\beta) H_{6}^{\beta}<-H_{6}^{\beta}+\beta H_{6}^{\beta}+(1-\beta) H_{6}^{\beta} \\
=0 .
\end{gathered}
$$

Therefore, $\frac{\partial \Omega_{0}}{\partial P_{T}}>0$. 
Thus, we have demonstrated that (36), (37), and (38) decrease as trade costs decline. Therefore, trade liberalization reduces inefficiency generated by SBC. Q.E.D. 